Review Article

\title{
Emerging Roles of Redox-Mediated Angiogenesis and Oxidative Stress in Dermatoses
}

\author{
Dehai Xian, ${ }^{1}$ Jing Song $\mathbb{D}^{2},{ }^{2}$ Lingyu Yang, ${ }^{2}$ Xia Xiong, ${ }^{2}$ Rui Lai, ${ }^{2}$ and Jianqiao Zhong ${ }^{2}$ \\ ${ }^{1}$ Department of Anatomy, Southwest Medical University, Luzhou 646000, China \\ ${ }^{2}$ Department of Dermatology, The Affiliated Hospital of Southwest Medical University, Luzhou 646000, China
}

Correspondence should be addressed to Jianqiao Zhong; zjq7632@hotmail.com

Dehai Xian and Jing Song contributed equally to this work.

Received 21 November 2018; Revised 14 February 2019; Accepted 14 March 2019; Published 16 April 2019

Academic Editor: Mithun Sinha

Copyright (C) 2019 Dehai Xian et al. This is an open access article distributed under the Creative Commons Attribution License, which permits unrestricted use, distribution, and reproduction in any medium, provided the original work is properly cited.

\begin{abstract}
Angiogenesis is the process of new vessel formation, which sprouts from preexisting vessels. This process is highly complex and primarily involves several key steps, including stimulation of endothelial cells by growth factors, degradation of the extracellular matrix by proteolytic enzymes, migration and proliferation of endothelial cells, and capillary tube formation. Currently, it is considered that multiple cytokines play a vital role in this process, which consist of proangiogenic factors (e.g., vascular endothelial growth factor, fibroblast growth factors, and angiopoietins) and antiangiogenic factors (e.g., endostatin, thrombospondin, and angiostatin). Angiogenesis is essential for most physiological events, such as body growth and development, tissue repair, and wound healing. However, uncontrolled neovascularization may contribute to angiogenic disorders. In physiological conditions, the above promoters and inhibitors function in a coordinated way to induce and sustain angiogenesis within a limited period of time. Conversely, the imbalance between proangiogenic and antiangiogenic factors could cause pathological angiogenesis and trigger several diseases. With insights into the molecular mechanisms of angiogenesis, increasing reports have shown that a close relationship exists between angiogenesis and oxidative stress (OS) in both physiological and pathological conditions. OS, an imbalance between prooxidant and antioxidant systems, is a cause and consequence of many vascular complains and serves as one of the biomarkers for these diseases. Furthermore, emerging evidence supports that OS and angiogenesis play vital roles in many dermatoses, such as psoriasis, atopic dermatitis, and skin tumor. This review summarizes recent findings on the role of OS as a trigger of angiogenesis in skin disorders, highlights newly identified mechanisms, and introduces the antiangiogenic and antioxidant therapeutic strategies.
\end{abstract}

\section{Introduction}

The complex process, regulated by proangiogenic and antiangiogenic factors, scientifically understood as the beginning formation of new blood vessels from existing ones, is known as angiogenesis [1]. New blood vessel formation, based on the balance of proangiogenic and antiangiogenic factors, is overwhelmingly responsible for most physiological events, such as embryogenesis, organ regeneration, body growth and development, skin renewal, and wound healing [2-4]. In the skin, angiogenesis is reactivated during skin renewal, wound healing, and tissue repair; furthermore, in these conditions, many angiogenic factors are released by activated keratinocytes and some inflammatory cells and jointly function to promote skin recovery and rejuvenation [5]; however, this process may be impaired by excessive angiogenic factors. In certain pathological conditions, these factors become overmuch and the balance between angiogenic promoters and inhibitors shifts, resulting in an angiogenic switch. The most well-known conditions where this switch is seen are malignant and inflammatory skin disorders as well as other pathological events, e.g., age-related macular degeneration, rheumatoid arthritis, tumor growth, proliferative retinopathies, and skin diseases (psoriasis, atopic dermatitis (AD), systemic sclerosis (SSc), cutaneous carcinoma, etc.) [5-8]. Either physiological or pathological angiogenesis is in need 
of initial mediation by various proangiogenic factors, consisting of endothelial growth factor (VEGF), fibroblast growth factors (FGF), interleukin-8 (IL-8), plateletderived growth factor (PDGF), placental growth factor (PGF), angiopoietin-1 (Ang-1), and transforming growth factor- $\beta$ (TGF- $\beta$ ) [9]. These proangiogenic factors subsequently induce a continuous recruitment of inflammatory cells to participate in the pathological process, which in turn serve as a substantial source of reactive oxygen species (ROS) [10, 11]. More importantly, excessive ROS trigger oxidative stress (OS), further promoting angiogenesis, damaging cells/tissue, and resulting in a variety of pathological changes [12].

OS is frequently considered as an imbalance of redox originating from the overproduction of prooxidants (e.g., ROS, reactive nitrogen species (RNS), nitric oxide (NO), and lipid peroxides) or from the insufficiency of antioxidants/antioxidant enzymes, like superoxide dismutase (SOD), catalase (CAT), and glutathione peroxidase (GPx) (Figure 1) $[13,14]$. Although the definition of OS is controversial, OS is currently regarded as a state in which stationary ROS/RNS transiently or gradually accumulate and ascend, further damaging cellular constituents and disturbing cellular metabolism [15]. Based on its intensity, OS is classified as basal OS (BOS), low-intensity OS (LOS), intermediate intensity OS (IOS), and high-intensity OS (HOS). OS, meanwhile, is categorized as mild OS (MOS), temperate OS (TOS), and severe OS (SOS) according to its degree [16]. In the process of OS, multiple redox signaling pathways are involved, primarily containing the mitogen-activated protein kinases/activator protein-1 (MAPK/AP-1), nuclear factor kappalight-chain-enhancer of activated $\mathrm{B}$ cells (NF- $\kappa \mathrm{B}$ ), Janus kinase-signal transducer and activator of transcription (JAK-STAT), nuclear factor erythroid 2-related factor (Nrf2), phosphatidylinositol-3-kinase/protein kinase B (PI3K/Akt), and Toll-like receptor- (TLR-) mediated signal transduction pathway [17]. Through these signaling pathways, OS mediates in physiological or pathological events. For example, MOS contribute to cell survival, whereas SOS may damage macromolecules (DNA, proteins, and lipids) and organelles (mitochondria and membranes), even the whole tissues (Figure 1) [18]. ROS, the major contributors to OS, including oxygen-centered radical species (superoxide anion $\left(\mathrm{O}_{2}{ }^{\bullet-}\right)$, hydroxyl radical $\left({ }^{\bullet} \mathrm{OH}\right)$, and peroxyl radical $\left(\mathrm{R}^{-} \mathrm{O}_{2}{ }^{\circ}\right)$ ) and nonradical compounds (ozone $\left(\mathrm{O}_{3}\right)$, hypochlorous acid $(\mathrm{HOCl})$, and hydrogen peroxide $\left(\mathrm{H}_{2} \mathrm{O}_{2}\right)$ ), are often generated by various categories of cells like endothelial cells (ECs), perivascular adipocytes, epithelial cells, smooth muscle cells, and adventitial fibroblasts [17]. In a physiological context, ROS have important roles in cell/tissue physiological processes including cell signaling, homeostasis, skin regeneration/renewal, and wound healing. In the skin in particular, ROS are mainly responsible for cell damage in the ageing process. ROS in low concentrations, nevertheless, participate in a substantial number of physiological cell redox signaling pathways to maintain redox equilibrium [19]; more importantly, ROS generated from immune cells are potently available for host defense [20]. As germicides or an important player in cellular signaling, they are also vital to wound healing and skin repair, while high-level ROS create a redox imbalance in the skin further causing severe "oxidative stress," eventually leading to DNA, cell, and tissue damage [21-27]. Several studies have demonstrated that in high concentration, $\mathrm{H}_{2} \mathrm{O}_{2}$ could induce endothelial injury; however, $\mathrm{H}_{2} \mathrm{O}_{2}$ in low concentration generally stimulates angiogenesis in wound healing and skin repair [28]. Accumulating evidence also supports that ROS as well ROS-mediated OS are involved in the process of physiological and pathological angiogenesis $[29,30]$ and closely implicated in the pathogenesis and exacerbation of angiogenesis-related diseases containing dermatoses, neurodegenerative disorders, cardiovascular diseases, and metabolic disorders [31-38]. In this review, we provide an overview of the current knowledge of the link between OS and angiogenesis and their roles in certain skin diseases as well as the emerging therapeutic strategies.

\section{Role of OS in Angiogenesis}

With further knowledge of angiogenesis, the pathogenesis of angiogenesis to some extent gradually becomes clear. It arrives at a consensus that ROS-mediated OS plays a crucial role in the development of angiogenesis. Moreover, two signal pathways of angiogenesis mediated by OS have been identified. One is the VEGF-dependent signaling pathway, while another is the VEGF-independent pathway $[10,39,40]$.

2.1. Generation of ROS in Angiogenesis. At present, it is demonstrated that ROS-mediated redox signaling has a central role in angiogenesis. ROS act as a double-edged sword in the vasculature. In a physiological process, ROS work as an important component of signaling events and play an important role in cellular differentiation and maintenance of homeostasis [41]. However, overproduction of ROS $\left(\mathrm{O}^{-2}\right.$ and $\mathrm{H}_{2} \mathrm{O}_{2}$ ) in turn contributes to neovascularization [21]. In this process, two endogenous ROS sources are mainly involved in the angiogenesis; one is nicotinamide adenine dinucleotide phosphate (NADPH) oxidase of the NOX family, and another is mitochondrial electron transport chain reactions [21, 31, 42, 43]. NADPH oxidase, a major source of ROS in ECs, generates $\mathrm{O}_{2}{ }^{--}$by transferring electrons from NADPH to oxygen. There are seven isoforms of NADPH oxidases expressed in mammals, namely, Nox1, Nox2 (previously gp91phox), Nox3, Nox4, Nox5, Duox1, and Duox2. This NADPH oxidase homologue consists of the following subunits: gp91phox (newly termed Nox2), p22phox, p40phox, p47phox, p67phox, and GTPase Rac1 [35, 44-46]. NADPH oxidase may be activated by diverse growth factors including VEGF, angiopoietin-1, ischemia, and hypoxia, and then, ROS derived from NADPH oxidase mediate in VEGFR-2 autophosphorylation [45, 47]. Apart from NADPH oxidase, ROS, the intracellular ROS in particular, were as well originated from the mitochondria. In the mitochondria, over $95 \%$ of oxygen consumed by cells affords water molecule production via redox reactions. But at complexes I and III in the transport chain, less than $4 \%$ of oxygen, which is reduced to superoxide anion instead of water, can generate OS $[31,45]$. 


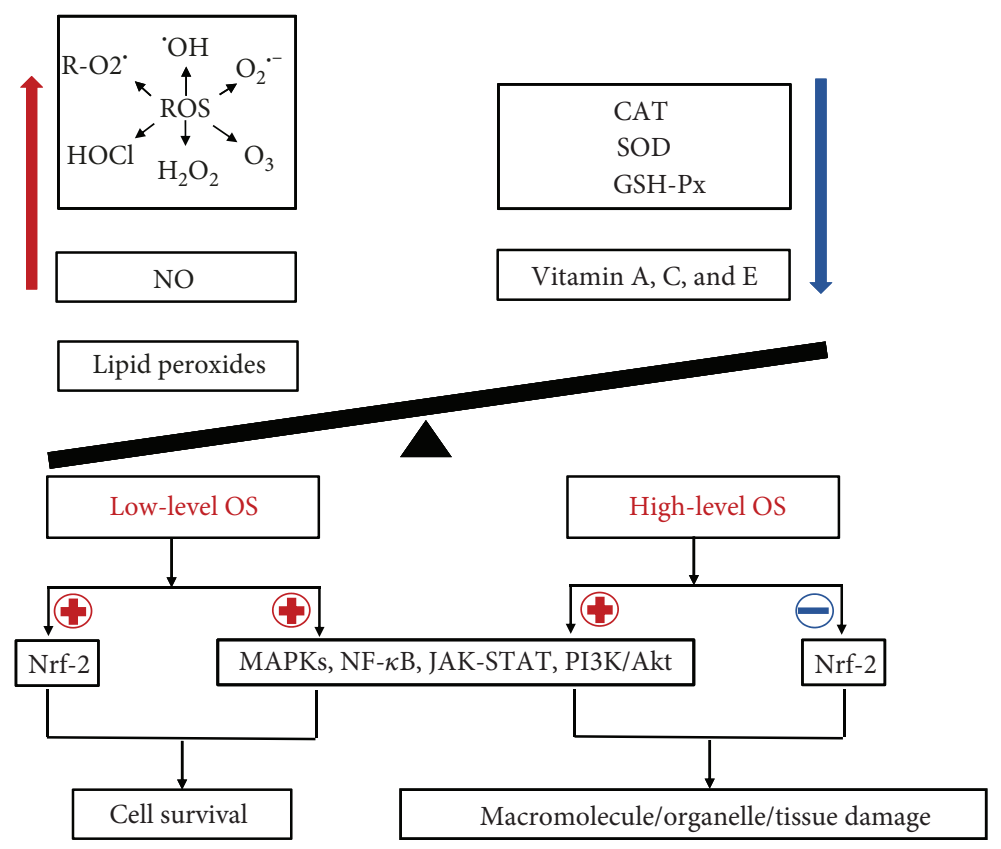

FIGURE 1: The process of oxidative stress (OS) generation. OS occurs when prooxidants (e.g., ROS, NO, and lipid peroxides) outbalance antioxidant defenses (e.g., SOD, CAT, and GSH-px). OS mediates in physiological or pathological events by activating/suppressing multiple redox signaling pathways (e.g., Nrf-2, MAPK, NF- $\kappa \mathrm{B}, \mathrm{PI} 3 \mathrm{~K} / \mathrm{Akt}$, and JAK-STA). For example, high-level OS may induce the damage of macromolecules (DNA, proteins, and lipids), organelles (mitochondria and membranes), and even the whole tissues, whereas low-level OS may contribute to cell survival. In this process, $\mathrm{ROS}$, including radical and nonradical $\mathrm{ROS}$ such as $\mathrm{O}_{2}{ }^{\bullet-},{ }^{\bullet} \mathrm{OH}, \mathrm{R}^{-} \mathrm{O}_{2}{ }^{\bullet}, \mathrm{O}^{3}$, $\mathrm{HOCl}$, and $\mathrm{H}_{2} \mathrm{O}_{2}$, play a pivotal role in the generation of OS. $\oplus$ means "to promote or enhance"; $\ominus$ means "to inhibit or suppress."

2.2. OS and Physiological Angiogenesis. Angiogenesis is physiologically essential for skin renewal, wound healing, tissue repair, skeletal remodeling, individual reproduction, etc. Among these physiological events, wound healing is a typical process involving angiogenesis and OS [20]. In this process, angiogenesis is induced by tissue hypoxia and ROS in either a VEGF-dependent way or a VEGF-independent way [48]. Low-concentration ROS facilitate angiogenesis in mouse wound healing and skin repair, which are involved in VEGF and its receptor signaling [49]. As the potent inducer of VEGF, hypoxia inducible factor 1 (HIF1) activated by ROS promotes angiogenesis via triggering VEGF expression during wound repair [50]. Thus, angiogenesis could be induced by ROS-mediated OS in a VEGF-dependent manner in wound healing. Increasing evidence has verified that VEGF can promote angiogenesis via binding to VEGF receptor-2 (VEGFR-2) in endothelial cells. The binding of VEGF to VEGFR-2 allows to activate a series of signal transduction molecules, including phospholipase C gamma (PLC $\gamma$ ) and phosphatidylinositol 3-kinase (PI3K), further stimulates the Raf-MAPK-ERK (mitogen-activated protein kinase/extracellular signal-regulated kinase) pathway, and finally facilitates angiogenesis $[51,52]$. It is therefore considered that VEGF/VEGFR-2 signaling is a crucial signal transducer in both physiologic and pathologic angiogenesis. Apart from VEGF, other soluble factors like PDGF play an important role in angiogenesis during wound healing, which is dependent on $\mathrm{H}_{2} \mathrm{O}_{2}$ for its biological function [53]. Upon activation of the PDGF pathway, signaling occurs via the PI3K/Akt complex pathway and MAPK molecules [54]. Besides, endogenous
2- $\omega$-carboxyethyl pyrrole (CEP), one major member of the carboxyalkyl pyrrole (CAP) family, is recognized by Tolllike receptor2 (TLR2) on endothelial cells and then activates MyD88-dependent signaling to promote angiogenesis at the wound site, which, in turn, accelerates wound healing. Consequently, OS also acts as the chief mediator of the VEGFindependent pathway in angiogenesis during the wound repair process [10].

2.3. OS and Pathological Angiogenesis. Pathological angiogenesis, fundamentally similar to physiological angiogenesis, is also affected by OS in VEGF-dependent and VEGFindependent ways, which proceeds in an unbalanced and uncontrolled fashion, finally resulting in an excessive and abnormal vascular pattern [55].

2.3.1. VEGF-Dependent Signaling Pathway. As one of the major angiogenesis factors, VEGF stimulates EC proliferation and migration via binding to VEGFR-2 regardless of physiological status or pathological condition, further activates several downstream signaling cascades, such as mitogen-activated protein kinases (MAPKs), PI3k/AKT, or eNOS, and eventually leads to physiological or pathological angiogenesis. Physiological angiogenesis like wound angiogenesis has been discussed before, and the mechanism of pathological angiogenesis is as follow.

The VEGF signal is essential for homeostasis and vascular development, which is always influenced by ROS [35]. Increasing evidence has indicated that most OS-related angiogenesis depends on VEGF involvement. Xia et al. 
showed that NADPH oxidase-dependent ROS stimulated VEGF secretion and facilitated excessive angiogenesis in a tumor microenvironment through the HIF-1 $\alpha$-mediated VEGF pathway, further promoting tumor growth [56]. ROS from follicle-stimulating hormone (FSH) triggered HIF- $1 \alpha$ signal and activated the VEGF signaling pathway by binding HIF- $1 \alpha$ to the VEGF promoter and further accelerated excessive angiogenesis and finally contributed to ovarian epithelial cancer progression [57]. Likewise, products of oxidation exemplified by oxidized phospholipids (OxPLs) stimulate VEGF expression both in vivo and in vitro, thereby interacting with VEGFR-2 and triggering angiogenesis [58]. Especially, oxidized low-density lipoproteins (OxLDL) originated from OS could strongly induce HIF-1a and VEGF expression in monocyte macrophages and significantly enhance tube formation in cocultured endothelial cells [5961]. As Toll-like receptor (TLR) ligands, poly (I:C) and lipopolysaccharide (LPS) both generated from OS, are also able to encourage angiogenesis via stimulating VEGF secretion or production and activate HIF- $1 \alpha$ and the TLR pathway in a TLR-dependent manner $[55,62]$. In addition, nitric oxide (NO) is considered to be one of the major contributors to angiogenesis and it has a capability of increasing the expression of HIF- $1 \alpha$ and VEGF, thereby leading to angiogenesis [63]. Thus, ROS-promoting angiogenesis is dependent on VEGF and the HIF- $1 \alpha /$ VEGF/VEGFR-2 pathway is a key molecular mechanism of OS-mediated angiogenesis [60].

2.3.2. VEGF-Independent Signaling Pathway. Apart from the VEGF-dependent pathway, another novel mechanism of OSmediated angiogenesis in a VEGF-independent manner recently has been demonstrated. Nowadays, because of some malignant tumors being resistant to anti-VEGF therapy, it is widely considered that the existence of VEGF-independent signaling is mainly responsible for this treatment-resistant event. In most cases, this resistance to anti-VEGF is linked with inflammation and infiltration of myeloid cells, which could create substantial oxygen tension and result in the accumulation of CEPs and finally accelerate neovascularization in a VEGF-independent manner [10]. There are two main VEGF-independent signaling pathways involved in angiogenesis, the CEP/TLR2/MyD88 axis and ROS/ataxiatelangiectasia mutated (ATM)/p38 $\alpha$ pathways $[10,64]$. The former mediates proangiogenesis and involves the accumulation of new lipid oxidation products, e.g., CAP protein adducts $[55,65]$. CEP acts as a potential biomarker for OSinduced vascular disorders and has the same proangiogenic effect as VEGF in vitro [66]. It has been demonstrated that TLRs not only serve as guardians of innate immunity but also function as prominent contributors to angiogenesis [67]. At present, it has been discovered that several proangiogenic ligands of TLRs produced by OS promote angiogenesis in a VEGF-independent way, such as CEP (a TLR2 ligand), macrophage-activating lipopeptide-2 (MALP-2) (a TLR2/6 ligand), and LPS (a TLR4 ligand). The molecular pattern of CEP, for example, is recognized by TLR2 on endothelial cells and triggers the MyD88-dependent signal to accelerate neovascularization [68]. LPS could stimulate endothelial sprouting directly in vitro through a TRAF6-mediated activation of
$\mathrm{NF}-\kappa \mathrm{B}$ and JNK [69]. Angiogenesis is also induced via GMCSF by TLR2/6 ligand binding to its receptor [70-72].

On the other hand, the latter, namely, ATM kinase, known for its function in the regulation of cell cycle and DNA damage repair, has been identified as an alternative mediator of OS-induced angiogenesis [73-75]. Remarkably, compared to CEP-TLR2 in angiogenesis, ATM in angiogenesis is uniquely limited to promoting the pathological process and ATM activation enhances no other cells but endothelial cell proliferation, which provides a probability for antiATM therapy [73]. As the downstream of ATM in endothelial cells, p38 is also involved in response to ROS; diminishing of ATM also suppressed angiogenesis even in the absence of VEGF inhibitors, suggesting a VEGF-independent proangiogenic role of ATM [73]. Figure 2 sketches two pathways of OS-mediated angiogenesis.

\section{OS and Angiogenesis in Dermatoses}

Growing evidence supports that OS and angiogenesis are both closely implicated in the occurrence and development of some skin diseases, such as psoriasis, AD, malignant melanoma (MM), Behcet's disease (BD), and scleroderma. However, the specific mechanism still remains unclear; thus, we concentrate on recent findings to present the possible mechanism of OS and angiogenesis in these cutaneous diseases.

3.1. OS and Angiogenesis Associated with Psoriasis. Psoriasis is a common chronic inflammatory skin disease approximately affecting $2 \%$ of the population. It characteristically manifests as erythema and papules/plaques accompanied by thick silvery-white scales. Nowadays, there is a wide range of options available for the treatment of psoriasis, such as topical therapies, phototherapy, older small-molecule systemic agents (e.g., methotrexate, cyclosporine, acitretin, and fumaric acid), the newer oral phosphodiesterase- 4 inhibitor apremilast, and the biologics (e.g., etanercept, adalimumab, infliximab, and ustekinumab) [76]. Despite that these therapies offer a certain efficacy, patients scarcely get satisfaction with substantial psoriatic lesion clearance, symptom relief, and improvements in quality of life [77]. Thus, there is a pressing need to develop some novel effective remedies. Although the etiology of psoriasis still remains unclear, it is thought that oxidative and angiogenic mechanisms both get involved in the pathological process of psoriasis. As one of major pathological features of psoriasis, angiogenesis has been persistently studied and various proangiogenic mediators have been identified in the psoriatic skin. Heidenreich et al. revealed a large spectrum of proangiogenic factors to mediate in psoriasis, including VEGF, HIF- $1 \alpha$, TNF, angiopoietins, IL-8, IL-17, and TGF- $\alpha$ [78].VEGF expression, in particular, remarkably elevated in the psoriatic serum and lesions. Moreover, ROS induced VEGF releasing from various cell types, whereas VEGF in turn promoted endothelial cell migration and proliferation through an increase of intracellular ROS. Thus, the VEGF pathway may be a crucial link between OS and angiogenesis in psoriasis, especially for the HIF-1 $\alpha$ /VEGF signaling pathway playing a synergistic role in the neovascularization of psoriasis $[79,80]$. By 


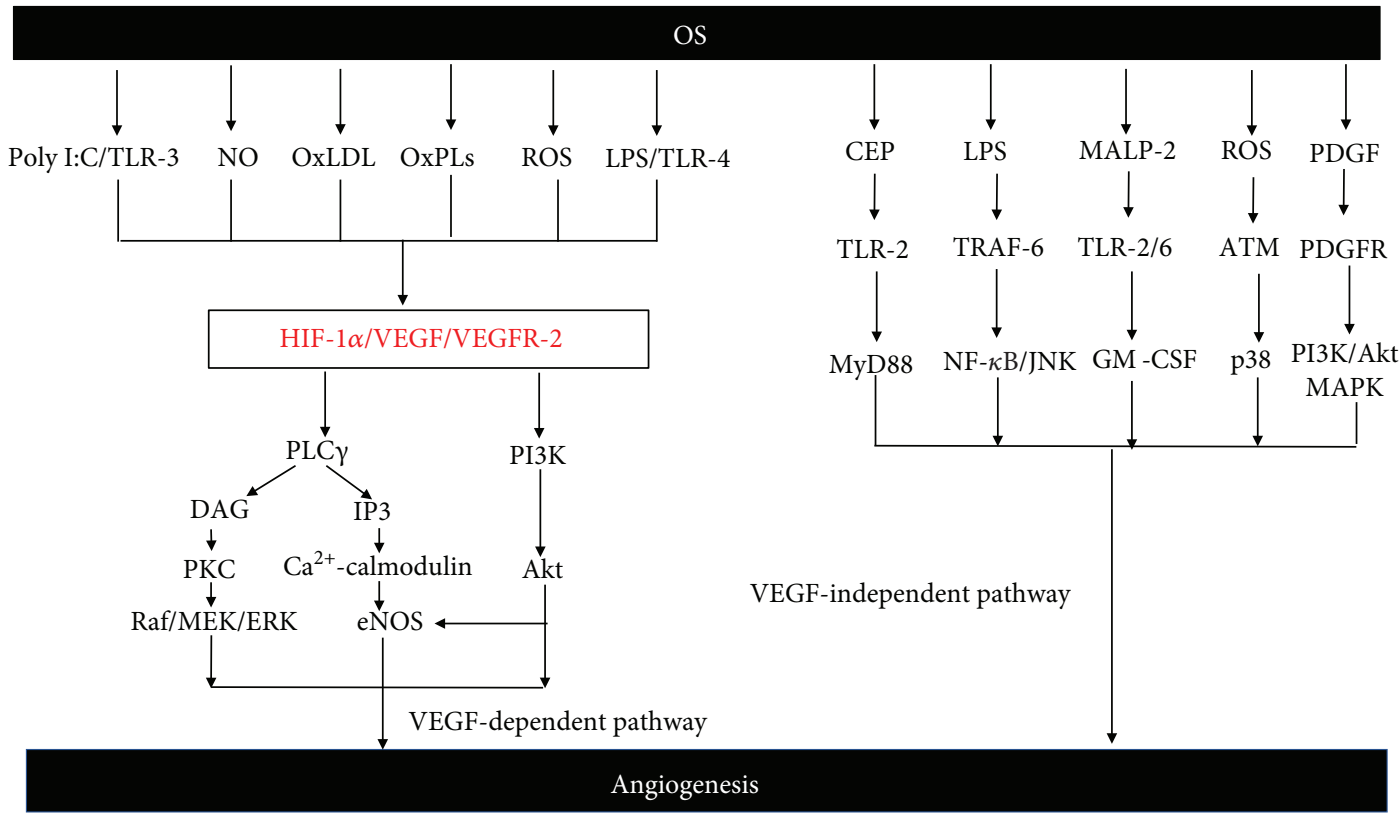

FIGURE 2: Schematic illustration of angiogenesis signaling pathways induced by OS. According to different responses to OS, two signal pathways of angiogenesis are covered, namely, the VEGF-dependent signaling pathway and VEGF-independent signaling pathway. In the VEGF-dependent pathway, ROS, NO, OxLDL, and OxPLs strongly stimulate the expression of HIF-1a and VEGF. Meanwhile, Poly I:C and LPS promote the expression of HIF- $1 \alpha$ and VFGF by coupling with their specific receptors (TLR3 and TLR4). These further combine to the downstream receptor VEGFR-2 and facilitate angiogenesis by activating the HIF-1 $\alpha$ /VFGF/VEGFR-2 signaling pathway in a VEGFdependent manner. On the other hand, many mediators are involved in the VEGF-independent pathway, including CEP, LPS, MALP-2, PDGF, and ROS. CEP/yyMALP-2 initially couples to their receptors (TLR2/6), then sensitizes specific downstream targets (e.g., MyD88 and GM-CSF), and finally promotes angiogenesis. Meanwhile, ROS activate the P38 pathway via inducing the activation of ATM and ultimately result in angiogenesis. Besides, LPS is considered to induce angiogenesis through a TRAF6-mediated activation of NF- $\kappa$ B and JNK. As another soluble mediator, PDGF triggers PI3K/Akt and MAPK signaling by binding to its receptor and promotes neovascularization.

upregulating the expression of cell adhesion molecules, VEGF could enhance the migration of leukocytes into the psoriatic skin and increase oxygen consumption, further activating HIF- $1 \alpha$ and perpetuating the angiogenic/inflammatory cycle of psoriasis [30, 81]. Furthermore, OxPLs afford the pathogenesis of psoriasis through enhancing VEGF generation from keratinocytes [30]. Besides, Elias et al. discovered that epidermal VEGF knockout mice scarcely appeared acanthosis after barrier disruption, suggesting an important contributor for VEGF to the development of keratinocyte hyperplasia. Hence, ROS-VEGF signaling may be a potential target for the treatment of psoriasis. However, the specific relationship between OS and angiogenesis in psoriasis requires to be further studied, which is conducive to fully clarify the pathogenesis of psoriasis and expand the optimal treatments for this disease.

3.2. OS and Angiogenesis Associated with $A D$. $\mathrm{AD}$, a chronic inflammatory skin disease, adversely affects many people especially young children [82]. The current management of $\mathrm{AD}$ covers avoidance of triggering factors, skin care, and anti-inflammatory therapy (mostly topical corticosteroids and topical calcineurin inhibitors). Once these first-line approaches are unsuccessful, systemic therapy or phototherapy tends to be carried out as second-line treatment [83]. After being treated with these vehicles, most symptoms may be relieved. However, long-term use of the above therapeutic probably causes many side effects such as skin atrophy and dryness, photoaging, and potential occurrence of cutaneous malignancies [84]. Therefore, some novel therapies are needed for the management of AD. The pathogenesis of AD is complex and still poorly understood. Recently, emerging evidence suggests that $O S$ is a potential key factor in the pathogenesis of $\mathrm{AD}$ [85]. OS is implicated in $\mathrm{AD}$ for several decades and remains present throughout the disease, including the onset of $\mathrm{AD}$, the development of $\mathrm{AD}$, and the exacerbation of AD. Moreover, excessive ROS overwhelm and destroy the skin antioxidant defense, which ultimately lead to $\mathrm{AD}$ progression and exacerbation [86]. Apart from OS, angiogenesis, as a hallmark of chronic inflammatory disorders, also gets involved in $\mathrm{AD}$ [87]. Several angiogenic factors contribute to the presence of angiogenic switch in the AD skin, such as VEGF, Angs, and IL-17. It has been demonstrated that angiogenesis is dysregulated in $\mathrm{AD}$ patients or models and high-level VEGF is detected in AD patient lesions. Meanwhile, Chen et al. discovered that a progressive increase of VEGF-A mRNA appeared in the skin of an AD mouse model [88]. Taken together, both OS and angiogenesis are mainly responsible for the pathogenesis of $\mathrm{AD}$ and the VEGF pathway may be a potential link between OS and angiogenesis in AD. Therefore, specific inhibitors targeting various mediators (e.g., VEGFs), receptors (e.g., VEGFRs and Tie-2), and oxides offer a promising foreground for the treatment of $\mathrm{AD}$ [7]. 
3.3. OS and Angiogenesis Associated with MM. MM, a malignant tumor of melanocytes, is accounted for about $10 \%$ of skin cancers, but it is responsible for over $90 \%$ of skin cancer deaths. For years, the cornerstones of cancer treatment have been surgery, chemotherapy, and radiation therapy. During the last decade, new strategies emerge from antitumor therapy for MM, including immunotherapy (e.g., checkpoint blockades) and targeted therapy (e.g., protein kinase inhibitors) or their combination [89]. Despite of extensive novel approaches serving for MM, the response rate is rarely higher than $20 \%$ and drug resistance is very common [90]. Up to date, rarely effective treatment has been approved for MM due to these reasons. As a result, it is urgent to invent other alternatives and targeted therapies [91, 92]. Compared to other tumors, MM is known for abundant ROS that exist in the primary tumor environment $[93,94]$. ROS from OS at one time had been recognized as a powerful weapon for the immune system to kill tumor cells [95]. However, once MM cells successfully escape ROS-induced apoptosis, persistent ROS tend to favor melanoma survival, proliferation, and metastasis through activating several related pathways [96]. Thus, ROS and ROS-mediated OS are closely implicated in different stages of MM. Elevated ROS could trigger the occurrence of OS, which further disrupt the homeostasis of melanocytes, affect the epigenetic regulation, and induce gene mutation, ultimately leading to cancer generation [97]. Accordingly, much efforts have been made to battle melanoma by using antioxidants so far [98]. Moreover, ROS and ROS-mediated OS would promote MM angiogenesis in a VEGF-dependent or VEGF-independent manner; in their publication, Bald et al. as well have emphasized the importance of the vascular network for MM [99]. Several angiogenic factors (e.g., VEGF, bFGF, PIGF, PDGF, IL-8, and Ang-1) have been found to highly express in primary skin $\mathrm{MM}$, and these mediators further promote MM angiogenesis and metastasis [100]. In addition, intratumoral hypoxia encourages the consequent expression of HIF- $\alpha$ transcription factors, in turn modulating VEGF and transcriptional product expression and mediating in cell growth, metabolism, and death [101, 102]. Meanwhile, preclinical studies indicate that the inhibitors targeting VEGF or VEGFR are effective in slowing the growth and metastasis of MM in murine models $[103,104]$. Apart from VEGF, PDGF and its receptor PDGFR- $\beta$ are responsible for MM angiogenesis. PDGF signaling is also implicated in angiogenesis in a VEGFindependent fashion. Therefore, OS and angiogenesis play vital roles in the development of MM; VEGF and PDGF signaling, moreover, may be the key link to OS and angiogenesis, which probably become the potential targets for the treatment of MM [105].

3.4. OS and Angiogenesis Associated with BD. BD, a chronic and recurrent vasculitis disease, is characterized by various clinical manifestations including skin lesions, oral/genital ulcer, ocular symptoms/lesions, joint signs, and organ involvements [106-108]. Glucocorticoids, azathioprine, cyclophosphamide, and cyclosporine A are currently the mainstay of treatments in vasculo-Behcet's disease, but long-term use of these drugs may induce some systemic adverse reactions [109]. Once immunosuppressive and corticosteroid therapies fail, biologic agents (e.g., infliximab, alemtuzumab, and adalimumab) can help for vascular lesions. However, high cost may be an obstacle to their widespread application [110]. Although BD etiology keeps being unknown, recently, growing evidence supports that elevated OS and insufficient antioxidant capacity are primarily involved in the pathogenesis of $\mathrm{BD}$ [111-113]. In the process of $\mathrm{BD}$ attack, ROS overproduction from OS may in turn accelerate OS aggression, then lead to tissue damage, and ultimately result in the pathological and clinical manifestations of BD. More importantly, we have demonstrated in our previous studies that there is an abnormal OS indeed existing in BD and a skewed redox balance remains present throughout this disease [114]. Apart from the OS-mediated mechanism, vascular endothelial activation is also considered to be a major one in $\mathrm{BD}$ [115-117]. Nowadays, it has been confirmed that several angiogenesis-promoting molecules (namely, angiogenic promoters) get involved in the pathogenesis of $\mathrm{BD}$, including IL-8, matrix metalloproteinases, Eselectin, vascular endothelial-cadherin, and VEGF [118]. Among them, VEGF, the dominant factor controlling angiogenesis, was found to highly express in BD serum and elevated-level VEGF was proportional to BD activity [119121]. VEGF, at the same time, plays an active role in the maintenance and growth of vascular endothelial cells. Kamoun et al. thought that high-level VEGF was closely associated with high concentration of $\mathrm{NO}$ from OS in BD [122]. Thus, OS and angiogenesis are crucial in BD pathogenesis and OS zealously mediates in the process of angiogenesis. However, further studies are needed to investigate the underlying mechanisms of OS-mediated angiogenesis in $\mathrm{BD}$, in order to develop new therapeutic strategies for $\mathrm{BD}$ patients to suppress OS and angiogenesis.

3.5. OS and Angiogenesis Associated with Scleroderma. Scleroderma, also known as systemic sclerosis (SSc), is a chronic immune-mediated connective tissue disease involving the skin, blood vessels, systemic organs, lungs, kidney, and gastrointestinal tract in particular [91]. SSc consists of two clinical subsets: one is limited cutaneous SSc (lc-SSc) and another is diffuse cutaneous SSc (dc$\mathrm{SSc}$ ). Because skin sclerosis can cause joint contracture, disability, and poor quality of life, various systemic treatments (e.g., penicillamine, cyclophosphamide, methotrexate, azathioprine, mycophenolate mofetil, intravenous immunoglobulin, and tyrosine kinase inhibitors) have been applied to alleviate the symptoms. These treatments, however, may cause severe side effects and offer inconsistent efficacy [123]. Phototherapy, another approach used to relieve skin sclerosis, provides a local effect on the skin without systemic involvement, but it alone cannot completely reverse skin sclerosis and it is just used as an adjunctive therapy together with other antifibrotic treatments (i.e., corticosteroids and pentoxifylline) [124]. Recently, it is thought that OS plays an important part in promoting scleroderma development, though SSc pathogenesis remains obscure [125]. Murrell proposed that an abnormal generation of ROS should be responsible for 
most of the pathologic features of SSc [126]. For example, ROS could stimulate the production of profibrotic cytokines (including PDGF and TGF- $\beta$ ) and proinflammatory factors, accelerate the activation and proliferation of fibroblasts, promote the synthesis of type I collagen, and induce vascular dysfunction [127]. By targeting ROS-generating NADPH oxidase, fibroblast activation and experimental skin fibrosis are inhibited in vitro and in vivo [128]. On the other hand, several abnormalities in regulating angiogenic responses in scleroderma indicate that aberrant angiogenesis may be another important pathogenic factor of scleroderma [129]. Hummers et al. have found that high levels of angiogenic factors were measured in patients with scleroderma, e.g., VEGF, PDGF-BB, FGF2, and PlGF [130]. Meanwhile, increased-level VEGF and VEGFR have been discovered in the serum and skin samples from scleroderma patients [131-135]. Besides, HIF-1a is more prevalent in SSc patients than normal subjects [136]. Accordingly, the pathogenesis of scleroderma is closely associated with OS and abnormal angiogenesis but further studies focused on the link between OS and angiogenesis in SSc are still needed, which may lead to the development of a new way for scleroderma treatment.

3.6. OS and Angiogenesis Associated with Rosacea. Rosacea is a common chronic inflammatory dermatosis, clinically characterized by erythema of the central face, episodic flushing, papules, and pustules [137, 138]. Skin care and pharmacologic treatments are the pillars of effective management of rosacea. Apart from existing topical agents (sodium sulfacetamide, azelaic acid, metronidazole, and the alphaadrenergic agonist brimonidine) and systemic medications (tetracyclines, beta-blockers and isotretinoin), new therapies including serine protease inhibitors and mast cell stabilizers may ameliorate rosacea symptoms [139]. However, some of these approaches have not been approved by the Food and Drug Administration. Though the exact pathogenesis of rosacea needs to be clarified, OS and oxidation of lipids are considered as crucial factors to trigger and aggravate the inflammatory processes of rosacea. Increased OS and decreased antioxidants are determined in systemic circulation of rosacea $[140,141]$. OS, in addition, is complicated in vascular changes, inflammation, and oxidative tissue damage in rosacea [142]. Therefore, antioxidants may be a potential strategy for treating rosacea. As an essential process in chronic inflammatory dermatoses, angiogenesis also contributes to the development of rosacea [143-145]. Amal et al. reported that VEGF expression elevated in cutaneous lesions of rosacea and was consistent with vascular histological changes which clinically presented as erythema and telangiectasia [146]. VEGF, indeed, has an important impact on the angiogenesis process, responsible for telangiectasia and increased vascular permeability, leading to cutaneous inflammation and the presence of papules, pustules, and nodules in rosacea $[147,148]$. Thus, attenuation of OS and VEGF may be relevant approaches for the therapy of rosacea. However, more research should be carried out to clarify the relationship of OS and angiogenesis and provide a novel therapeutic way for rosacea.

\section{Therapeutic Implications}

Given that OS and OS-mediated angiogenesis have important roles in promoting various dermatoses, it should be fully suitable to develop novel therapies for skin disorders aimed at both aspects (Figure 3). As a major regulator of angiogenesis, VEGF and its pathway are considered as key targets for antiangiogenic therapy $[149,150]$. Some effective drugs targeting VEGF have emerged from the pharmaceutical industry to inhibit new vessel formation.

4.1. Antiangiogenic Agents in the Management of Skin Diseases. Based on successful phase III trials, antiangiogenic therapeutics, anti-VEGF agents in particular (e.g., sorafenib, bevacizumab, and sunitinib), have entered the clinical practice in the USA and elsewhere. Strategies have been developed to inhibit the VEGF signaling pathway including antiVEGF antibody therapy (e.g., bevacizumab), anti-VEGFR antibody therapy (e.g., ramucirumab), inhibitors of VEGFR-2 tyrosine kinases (e.g., apatinib), and inhibitors of angiogenic receptor tyrosine kinases (e.g., sunitinib, pazopanib, sorafenib, and regorafenib) [151]. Due to their antiangiogenic, antioxidative, and antiproliferative effects, phytochemicals are beneficial in the battle against cutaneous carcinoma [152]. Intraperitoneal injection of recombinant thrombospondin type 1 repeat domain (rTSR1) or a disintegrin-like and metalloproteinase with thrombospondin motifs 5 (ADAMTS5) could potently inhibit subcutaneous melanoma growth by diminishing angiogenesis, promoting apoptosis, and decreasing cell proliferation in the tumor tissue [153]. Antiangiogenic agent AE-941 from extracts of cartilage potentially provides a beneficial effect to treat cutaneous and systemic diseases especially for psoriasis [154]. It is speculated that cannabinoids have a potential role in treatment of psoriasis by controlling angiogenesis and inflammation through decreasing HIF- $1 \alpha$ and VEGF levels [155]. Meanwhile, Kuang et al. also demonstrated that topical sunitinib ointment contributed to attenuate imiquimodinduced psoriasis-like inflammation through regulating the proliferation and apoptosis of keratinocytes via suppressing p-Stat3 and VEGF expression [156]. Besides, thalidomide effectively works in skin disorders such as BD through inhibition of VEGF- and FGF-2-mediated angiogenesis [157].

\subsection{Agents against OS in the Management of Dermatoses. On} the other side, it is quite beneficial to skin disorder recovery by employing OS-targeted drugs like antioxidants. Because OS-dependent angiogenesis is an important contributor to the progression of cancers, antioxidants may overcome the limitations of anti-VEGF therapy, especially in relation to tumor resistance. Related documents revealed that glabridin ameliorated imiquimod-induced psoriasis-like inflammation on $\mathrm{BALB} / \mathrm{c}$ mice skin through improvement of antioxidant status and downregulation of proinflammatory cytokines [158]. By decreasing lipid peroxidation and modulating $\mathrm{Ca}^{2+}$ release, colchicine significantly induced protective effects on OS in the neutrophils of BD patients [159]. More importantly, high-dose vitamin C could effectively work in the skin diseases of $\mathrm{MM}$ and $\mathrm{AD}$ owing to its antioxidant 


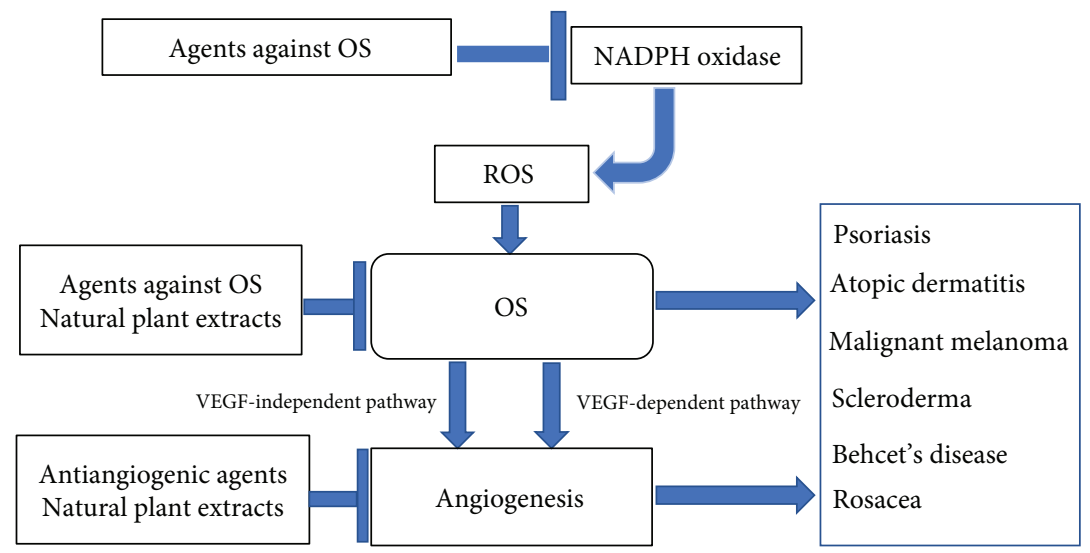

FIGURE 3: Strategies for dermatoses through mediating OS-induced angiogenesis and OS. Several novel therapies used for OS or angiogenesisrelated skin disorders have been developed, including antiangiogenic agents, agents against OS, and natural plant extracts. These vehicles are potentially effective in management of dermatoses (e.g., psoriasis, $\mathrm{AD}, \mathrm{MM}$, scleroderma, $\mathrm{BD}$, and rosacea) through mediating OS or angiogenesis-associated signal pathways.

protection [160]. Apart from the traditional antioxidants, NADPH oxidase, a key enzyme generation of ROS in neovascularization, potentially becomes the important target of pharmacological inhibitors. And NOX inhibitors are the most promising therapeutic option for diseases associated with OS. Among them, traditional NADPH oxidase inhibitors, such as apocynin and diphenylene iodonium, have no specificity and little isoform selectivity. Instead, several novel NOX inhibitors (GKT137831, ML171, and VAS2870) exhibit improved specificity for NADPH oxidases and NOX isoform selectivity [161].

4.3. Natural Plant Extracts in the Management of Dermatoses. Nowadays, natural extracts from plants increasingly arrest the attention from medical fields and pharmaceutical industry. Numerous natural extracts, like tea polyphenol, proanthocyanidins, and allicin, are potently beneficial to various skin disorders. As the main active ingredient of tea polyphenol, epigallocatechin-3-gallate (EGCG) could prevent OS-induced damage and suppress angiogenesis to avail against skin cancer and psoriasis basing on its antioxidant, antitumor, and antiangiogenic properties [162, 163]. Due to their powerful antioxidation, antiangiogenesis, antiproliferation, and antioncogenesis, proanthocyanidins have a wide utilization in the management of various OS-related and angiogenic complaints $[164,165]$. Phenolic metabolites [166]. Moreover, we have proposed in our previous publications that proanthocyanidins are good for the treatment of psoriasis, AD, allergic purpura, SSc, rosacea, skin cancer, and other dermatoses [167]. Besides, our recent finding reveals that allicin, an active substance from garlic, has a favorable efficacy on BD by attenuation of OS and balance of oxidant/antioxidant status [168].

\section{Conclusion}

In summary, there are two main mechanisms implicated in the area bridging angiogenesis and OS; one is the VEGFdependent signaling pathway (HIF/VEGF signaling), while another is the VEGF-independent signaling pathway (CEP/TLR2/MyD88 axis and ROS/ATM/p38 $\alpha$ pathway). It is clear that OS and OS-derived angiogenesis are important contributors to the progression of chronic diseases and tumors. There is no doubt that both OS and angiogenesis participate in the development of certain skin diseases; however, a deeper understanding of the mechanisms behind OS and OS-dependent angiogenesis is necessary. There is a need for an investigation of multifaceted pathways involved in OSinduced angiogenesis in dermatoses and a specific target discriminating pathological vasculature from the physiological one. Therefore, in addition to the anti-VEGF drugs and OS inhibitors or antioxidants, it is necessary to develop some newly specific target strategies.

\section{Conflicts of Interest}

No financial or other conflicting interest exists.

\section{Authors' Contributions}

Dehai Xian and Jing Song contributed equally to this work.

\section{Acknowledgments}

The authors thank Professor Liao Li and Yang Xu for their very helpful comments on this manuscript, which is greatly beneficial to the improvement of this paper.

\section{References}

[1] L. S. Rosen, "Clinical experience with angiogenesis signaling inhibitors: focus on vascular endothelial growth factor (VEGF) blockers," Cancer Control, vol. 9, 2 Supplement, pp. 36-44, 2002.

[2] A. Hoeben, B. Landuyt, M. S. Highley, H. Wildiers, A. T. Van Oosterom, and E. A. De Bruijn, "Vascular endothelial growth factor and angiogenesis," Pharmacological Reviews, vol. 56, no. 4, pp. 549-580, 2004. 
[3] A. Adini, I. Adini, Z.-l. Chi et al., "A novel strategy to enhance angiogenesis in vivo using the small VEGFbinding peptide PR1P," Angiogenesis, vol. 20, no. 3, pp. 399-408, 2017.

[4] S. Sajib, F. T. Zahra, M. S. Lionakis, N. A. German, and C. M. Mikelis, "Mechanisms of angiogenesis in microbe-regulated inflammatory and neoplastic conditions," Angiogenesis, vol. 21, no. 1, pp. 1-14, 2018.

[5] F. Granata, A. Frattini, S. Loffredo et al., "Production of vascular endothelial growth factors from human lung macrophages induced by group IIA and group $\mathrm{X}$ secreted phospholipases $\mathrm{A}_{2}$," The Journal of Immunology, vol. 184, no. 9, pp. 5232-5241, 2010.

[6] C. Viallard and B. Larrivée, "Tumor angiogenesis and vascular normalization: alternative therapeutic targets," Angiogenesis, vol. 20, no. 4, pp. 409-426, 2017.

[7] A. Genovese, A. Detoraki, F. Granata, M. R. Galdiero, G. Spadaro, and G. Marone, "Angiogenesis, lymphangiogenesis and atopic dermatitis," Chemical Immunology and Allergy, vol. 96, pp. 50-60, 2012.

[8] N. A. Richarz, A. Boada, and J. M. Carrascosa, "Angiogenesis in dermatology - insights of molecular mechanisms and latest developments," Actas Dermo-Sifiliográficas, vol. 108, no. 6, pp. 515-523, 2017.

[9] A. S. Chung and N. Ferrara, "Developmental and pathological angiogenesis," Annual Review of Cell and Developmental Biology, vol. 27, no. 1, pp. 563-584, 2011.

[10] Y. W. Kim, X. Z. West, and T. V. Byzova, "Inflammation and oxidative stress in angiogenesis and vascular disease," Journal of Molecular Medicine, vol. 91, no. 3, pp. 323-328, 2013.

[11] S. Schreml, R. M. Szeimies, L. Prantl, S. Karrer, M. Landthaler, and P. Babilas, "Oxygen in acute and chronic wound healing," British Journal of Dermatology, vol. 163, no. 2, pp. 257-268, 2010.

[12] S. Reuter, S. C. Gupta, M. M. Chaturvedi, and B. B. Aggarwal, "Oxidative stress, inflammation, and cancer: how are they linked?," Free Radical Biology \& Medicine, vol. 49, no. 11, pp. 1603-1616, 2010.

[13] M. Long, S. H. Yang, J. X. Han et al., "The protective effect of grape-seed proanthocyanidin extract on oxidative damage induced by zearalenone in Kunming mice liver," International Journal of Molecular Sciences, vol. 17, no. 6, p. 808, 2016.

[14] T. Finkel and N. J. Holbrook, "Oxidants, oxidative stress and the biology of ageing," Nature, vol. 408, no. 6809, pp. 239247, 2000.

[15] V. I. Lushchak, "Environmentally induced oxidative stress in aquatic animals," Aquatic Toxicology, vol. 101, no. 1, pp. 13$30,2011$.

[16] V. I. Lushchak, "Free radicals, reactive oxygen species, oxidative stress and its classification," Chemico-Biological Interactions, vol. 224, pp. 164-175, 2014.

[17] S. O. Yoon, C. H. Yun, and A. S. Chung, "Dose effect of oxidative stress on signal transduction in aging," Mechanisms of Ageing and Development, vol. 123, no. 12, pp. 1597-1604, 2002.

[18] Y. Han, C. Huang, X. Sun et al., "SENP3-mediated deconjugation of SUMO2/3 from promyelocytic leukemia is correlated with accelerated cell proliferation under mild oxidative stress," Journal of Biological Chemistry, vol. 285, no. 17, pp. 12906-12915, 2010.
[19] K. Bedard and K. H. Krause, "The NOX family of ROSgenerating NADPH oxidases: physiology and pathophysiology," Physiological Reviews, vol. 87, no. 1, pp. 245-313, 2007.

[20] T. Inoguchi, T. Sonta, H. Tsubouchi et al., "Protein kinase Cdependent increase in reactive oxygen species (ROS) production in vascular tissues of diabetes: role of vascular $\mathrm{NAD}(\mathrm{P}) \mathrm{H}$ oxidase," Journal of the American Society of Nephrology, vol. 14, Supplement 3, pp. S227-S232, 2003.

[21] R. M. Touyz and A. M. Briones, "Reactive oxygen species and vascular biology: implications in human hypertension," Hypertension Research, vol. 34, no. 1, pp. 5-14, 2011.

[22] N. Li, K. Ragheb, G. Lawler et al., "Mitochondrial complex I inhibitor rotenone induces apoptosis through enhancing mitochondrial reactive oxygen species production," Journal of Biological Chemistry, vol. 278, no. 10, pp. 8516-8525, 2003.

[23] A. Kanayama and Y. Miyamoto, "Apoptosis triggered by phagocytosis-related oxidative stress through FLIPS downregulation and JNK activation," Journal of Leukocyte Biology, vol. 82, no. 5, pp. 1344-1352, 2007.

[24] A. Gorlach, T. Kietzmann, and J. Hess, "Redox signaling through NADPH oxidases: involvement in vascular proliferation and coagulation," Annals of the New York Academy of Sciences, vol. 973, no. 1, pp. 505-507, 2002.

[25] P. Chiarugi, G. Pani, E. Giannoni et al., "Reactive oxygen species as essential mediators of cell adhesion," The Journal of Cell Biology, vol. 161, no. 5, pp. 933-944, 2003.

[26] V. J. Thannickal and B. L. Fanburg, "Reactive oxygen species in cell signaling," American Journal of Physiology-Lung Cellular and Molecular Physiology, vol. 279, no. 6, pp. L1005L1028, 2000.

[27] S. van Wetering, J. D. van Buul, S. Quik et al., "Reactive oxygen species mediate Rac-induced loss of cell-cell adhesion in primary human endothelial cells," Journal of Cell Science, vol. 115, Part 9, pp. 1837-1846, 2002.

[28] A. San Martin and K. K. Griendling, "Redox control of vascular smooth muscle migration," Antioxidants \& Redox Signaling, vol. 12, no. 5, pp. 625-640, 2010.

[29] P. Mu, Q. Liu, and R. Zheng, "Biphasic regulation of $\mathrm{H} 2 \mathrm{O} 2$ on angiogenesis implicated NADPH oxidase," Cell Biology International, vol. 34, no. 10, pp. 1013-1020, 2010.

[30] A. W. Armstrong, S. V. Voyles, E. J. Armstrong, E. N. Fuller, and J. C. Rutledge, "Angiogenesis and oxidative stress: common mechanisms linking psoriasis with atherosclerosis," Journal of Dermatological Science, vol. 63, no. 1, pp. 1-9, 2011.

[31] M. Ushio-Fukai and R. W. Alexander, "Reactive oxygen species as mediators of angiogenesis signaling. Role of $N A D(P) H$ oxidase," Molecular and Cellular Biochemistry, vol. 264, no. 1/2, pp. 85-97, 2004.

[32] L. Yang, D. Xian, X. Xiong, R. Lai, J. Song, and J. Zhong, "Proanthocyanidins against oxidative stress: from molecular mechanisms to clinical applications," BioMed Research International, vol. 2018, Article ID 8584136, 11 pages, 2018.

[33] U. auf dem Keller, A. Kumin, S. Braun, and S. Werner, "Reactive oxygen species and their detoxification in healing skin wounds," Journal of Investigative Dermatology Symposium Proceedings, vol. 11, no. 1, pp. 106-111, 2006.

[34] M. Ushio-Fukai, "Redox signaling in angiogenesis: role of NADPH oxidase," Cardiovascular Research, vol. 71, no. 2, pp. 226-235, 2006. 
[35] M. Ushio-Fukai and Y. Nakamura, "Reactive oxygen species and angiogenesis: NADPH oxidase as target for cancer therapy," Cancer Letters, vol. 266, no. 1, pp. 37-52, 2008.

[36] G. Waris and H. Ahsan, "Reactive oxygen species: role in the development of cancer and various chronic conditions," Journal of Carcinogenesis, vol. 5, no. 1, p. 14, 2006.

[37] W. Zhao, T. Zhao, Y. Chen, R. A. Ahokas, and Y. Sun, "Reactive oxygen species promote angiogenesis in the infarcted rat heart," International Journal of Experimental Pathology, vol. 90, no. 6, pp. 621-629, 2009.

[38] N. Maulik and D. K. Das, "Redox signaling in vascular angiogenesis," Free Radical Biology \& Medicine, vol. 33, no. 8, pp. 1047-1060, 2002.

[39] R. Colavitti, G. Pani, B. Bedogni et al., "Reactive oxygen species as downstream mediators of angiogenic signaling by vascular endothelial growth factor receptor-2/KDR," Journal of Biological Chemistry, vol. 277, no. 5, pp. 31013108, 2002.

[40] N. L. Malinin, X. Z. West, and T. V. Byzova, "Oxidation as "the stress of life"," Aging, vol. 3, no. 9, pp. 906-910, 2011.

[41] C. R. Reczek and N. S. Chandel, "ROS-dependent signal transduction," Current Opinion in Cell Biology, vol. 33, pp. 8-13, 2015.

[42] N. Maulik, "Redox regulation of vascular angiogenesis," Antioxidants \& Redox Signaling, vol. 4, no. 5, pp. 783-784, 2002.

[43] I. Takac, K. Schröder, and R. P. Brandes, "The Nox family of NADPH oxidases: friend or foe of the vascular system?," Current Hypertension Reports, vol. 14, no. 1, pp. 70-78, 2012.

[44] J. D. Lambeth, "NOX enzymes and the biology of reactive oxygen," Nature Reviews Immunology, vol. 4, no. 3, pp. 181-189, 2004.

[45] M. D. Brand, C. Affourtit, T. C. Esteves et al., "Mitochondrial superoxide: production, biological effects, and activation of uncoupling proteins," Free Radical Biology \& Medicine, vol. 37, no. 6, pp. 755-767, 2004.

[46] S. Coso, I. Harrison, C. B. Harrison et al., "NADPH oxidases as regulators of tumor angiogenesis: current and emerging concepts," Antioxidants \& Redox Signaling, vol. 16, no. 11, pp. 1229-1247, 2012.

[47] B. Lassègue, A. San Martín, and K. K. Griendling, "Biochemistry, physiology, and pathophysiology of NADPH oxidases in the cardiovascular system," Circulation Research, vol. 110, no. 10, pp. 1364-1390, 2012.

[48] I. T. Nizamutdinova, Y. M. Kim, J. I. Chung et al., “Anthocyanins from black soybean seed coats stimulate wound healing in fibroblasts and keratinocytes and prevent inflammation in endothelial cells," Food and Chemical Toxicology, vol. 47, no. 11, pp. 2806-2812, 2009.

[49] S. Roy, S. Khanna, K. Nallu, T. K. Hunt, and C. K. Sen, "Dermal wound healing is subject to redox control," Molecular Therapy, vol. 13, no. 1, pp. 211-220, 2006.

[50] Y. Heun, K. Pogoda, M. Anton et al., "HIF-1 $\alpha$ dependent wound healing angiogenesis in vivo can be controlled by site-specific lentiviral magnetic targeting of SHP-2," Molecular Therapy, vol. 25, no. 7, pp. 1616-1627, 2017.

[51] J. Bosch, J. G. Abraldes, M. Fernández, and J. C. GarcíaPagán, "Hepatic endothelial dysfunction and abnormal angiogenesis: new targets in the treatment of portal hypertension," Journal of Hepatology, vol. 53, no. 3, pp. 558-567, 2010 .
[52] D. Guo, C. E. Murdoch, T. Liu et al., "Therapeutic angiogenesis of Chinese herbal medicines in ischemic heart disease: a review," Frontiers in Pharmacology, vol. 9, p. 428, 2018.

[53] H. Lei and A. Kazlauskas, "Detection of $\mathrm{H}_{2} \mathrm{O}_{2}$-mediated phosphorylation of kinase-inactive PDGFR $\alpha$," Methods in Enzymology, vol. 528, pp. 189-194, 2013.

[54] Y. Xiao, H. Peng, C. Hong et al., "PDGF promotes the Warburg effect in pulmonary arterial smooth muscle cells via activation of the PI3K/AKT/mTOR/HIF- $1 \alpha$ signaling pathway," Cellular Physiology and Biochemistry, vol. 42, no. 4, pp. 16031613, 2017.

[55] R. G. Salomon, "Structural identification and cardiovascular activities of oxidized phospholipids," Circulation Research, vol. 111, no. 7, pp. 930-946, 2012.

[56] C. Xia, Q. Meng, L. Z. Liu, Y. Rojanasakul, X. R. Wang, and B. H. Jiang, "Reactive oxygen species regulate angiogenesis and tumor growth through vascular endothelial growth factor," Cancer Research, vol. 67, no. 22, pp. 10823-10830, 2007.

[57] Z. Zhang, Q. Wang, J. Ma et al., "Reactive oxygen species regulate FSH-induced expression of vascular endothelial growth factor via NRF2 and HIF1 $\alpha$ signaling in human epithelial ovarian cancer," Oncology Reports, vol. 29, no. 4, pp. 14291434, 2013.

[58] A. A. Birukova, S. Lee, V. Starosta et al., "A role for VEGFR2 activation in endothelial responses caused by barrier disruptive OxPAPC concentrations," PLoS One, vol. 7, no. 1, 2012.

[59] L. Salomonsson, S. Pettersson, M. C. O. Englund, O. Wiklund, and B. G. Ohlsson, "Post-transcriptional regulation of VEGF expression by oxidised LDL in human macrophages," European Journal of Clinical Investigation, vol. 32, no. 10, pp. 767-774, 2002.

[60] R. Hutter, W. S. Speidl, C. Valdiviezo et al., "macrophages transmit potent proangiogenic effects of oxLDL in vitro and in vivo involving HIF-1 $\alpha$ Activation: a novel aspect of angiogenesis in atherosclerosis," Journal of Cardiovascular Translational Research, vol. 6, no. 4, pp. 558-569, 2013.

[61] V. A. Shatrov, V. V. Sumbayev, J. Zhou, and B. Brüne, "Oxidized low-density lipoprotein (oxLDL) triggers hypoxiainducible factor- $1 \alpha$ (HIF- $1 \alpha$ ) accumulation via redoxdependent mechanisms," Blood, vol. 101, no. 12, pp. 48474849, 2003.

[62] A. Paone, R. Galli, C. Gabellini et al., "Toll-like receptor 3 regulates angiogenesis and apoptosis in prostate cancer cell lines through hypoxia-inducible factor $1 \alpha$," Neoplasia, vol. 12, no. 7, pp. 539-549, 2010.

[63] X. Lu and G. S. Kassab, "Nitric oxide is significantly reduced in ex vivo porcine arteries during reverse flow because of increased superoxide production," The Journal of Physiology, vol. 561, no. 2, pp. 575-582, 2004.

[64] Y. W. Kim and T. V. Byzova, "Oxidative stress in angiogenesis and vascular disease," Blood, vol. 123, no. 5, pp. 625-631, 2014.

[65] J. W. Crabb, M. Miyagi, X. Gu et al., "Drusen proteome analysis: an approach to the etiology of age-related macular degeneration," Proceedings of the National Academy of Sciences of the United States of America, vol. 99, no. 23, pp. 14682-14687, 2002.

[66] Q. Ebrahem, K. Renganathan, J. Sears et al., "Carboxyethylpyrrole oxidative protein modifications stimulate neovascularization: implications for age-related macular degeneration," Proceedings of the National Academy of 
Sciences of the United States of America, vol. 103, no. 36, pp. 13480-13484, 2006.

[67] Y. Bordon, "A new vein of TLR biology," Nature Reviews Immunology, vol. 10, no. 11, p. 748, 2010.

[68] X. Z. West, N. L. Malinin, A. A. Merkulova et al., "Oxidative stress induces angiogenesis by activating TLR2 with novel endogenous ligands," Nature, vol. 467, no. 7318 , pp. $972-$ 976, 2010.

[69] I. Pollet, C. J. Opina, C. Zimmerman, K. G. Leong, F. Wong, and A. Karsan, "Bacterial lipopolysaccharide directly induces angiogenesis through TRAF6-mediated activation of NF- $\kappa \mathrm{B}$ and c-Jun N-terminal kinase," Blood, vol. 102, no. 5, pp. 1740-1742, 2003.

[70] K. Grote, H. Schuett, G. Salguero et al., “Toll-like receptor 2/6 stimulation promotes angiogenesis via GM-CSF as a potential strategy for immune defense and tissue regeneration," Blood, vol. 115, no. 12, pp. 2543-2552, 2010.

[71] N. W. Palm and R. Medzhitov, "Pattern recognition receptors and control of adaptive immunity," Immunological Reviews, vol. 227, no. 1, pp. 221-233, 2009.

[72] T. Kawai and S. Akira, "Pathogen recognition with Toll-like receptors," Current Opinion in Immunology, vol. 17, no. 4, pp. 338-344, 2005.

[73] Y. Okuno, A. Nakamura-Ishizu, K. Otsu, T. Suda, and Y. Kubota, "Pathological neoangiogenesis depends on oxidative stress regulation by ATM," Nature Medicine, vol. 18, no. 8, pp. 1208-1216, 2012.

[74] Z. Guo, S. Kozlov, M. F. Lavin, M. D. Person, and T. T. Paull, "ATM activation by oxidative stress," Science, vol. 330, no. 6003 , pp. $517-521,2010$.

[75] C. J. Bakkenist and M. B. Kastan, "DNA damage activates ATM through intermolecular autophosphorylation and dimer dissociation," Nature, vol. 421, no. 6922, pp. 499506, 2003.

[76] B. Ladizinski, K. C. Lee, E. Wilmer, A. Alavi, N. Mistry, and R. G. Sibbald, "A review of the clinical variants and the management of psoriasis," Advances in Skin \& Wound Care, vol. 26, no. 6, pp. 271-284, 2013.

[77] C. Baker, A. Mack, A. Cooper et al., "Treatment goals for moderate to severe psoriasis: an Australian consensus," Australasian Journal of Dermatology, vol. 54, no. 2, pp. 148-154, 2013.

[78] R. Heidenreich, M. Röcken, and K. Ghoreschi, “Angiogenesis drives psoriasis pathogenesis," International Journal of Experimental Pathology, vol. 90, no. 3, pp. 232-248, 2009.

[79] A. Campanati, G. Goteri, O. Simonetti et al., "Angiogenesis in psoriatic skin and its modifications after administration of etanercept: videocapillaroscopic, histological and immunohistochemical evaluation," International Journal of Immunopathology and Pharmacology, vol. 22, no. 2, pp. 371-377, 2009.

[80] A. Nofal, I. Al-Makhzangy, E. Attwa, A. Nassar, and A. Abdalmoati, "Vascular endothelial growth factor in psoriasis: an indicator of disease severity and control," Journal of the European Academy of Dermatology and Venereology, vol. 23, no. 7, pp. 803-806, 2009.

[81] M. Detmar, L. F. Brown, M. P. Schön et al., "Increased microvascular density and enhanced leukocyte rolling and adhesion in the skin of VEGF transgenic mice," Journal of Investigative Dermatology, vol. 111, no. 1, pp. 1-6, 1998.
[82] R. Sidbury, D. M. Davis, D. E. Cohen et al., "Guidelines of care for the management of atopic dermatitis: section 3. Management and treatment with phototherapy and systemic agents," Journal of the American Academy of Dermatology, vol. 71, no. 2, pp. 327-349, 2014.

[83] J. M. Ortiz-Salvador and A. Pérez-Ferriols, "Phototherapy in atopic dermatitis," in Ultraviolet Light in Human Health, Diseases and Environment, vol. 996 of Advances in Experimental Medicine and Biology, pp. 279-286, Springer, Cham, Switzerland, 2017.

[84] D. L. Rodenbeck, J. I. Silverberg, and N. B. Silverberg, "Phototherapy for atopic dermatitis," Clinics in Dermatology, vol. 34, no. 5, pp. 607-613, 2016.

[85] N. Omata, H. Tsukahara, S. Ito et al., "Increased oxidative stress in childhood atopic dermatitis," Life Sciences, vol. 69, no. 2, pp. 223-228, 2001.

[86] H. Ji and X. K. Li, "Oxidative stress in atopic dermatitis," Oxidative Medicine and Cellular Longevity, vol. 2016, Article ID 2721469, 8 pages, 2016.

[87] A. Nguyen, V. Hoang, V. Laquer, and K. M. Kelly, "Angiogenesis in cutaneous disease: part I," Journal of the American Academy of Dermatology, vol. 61, no. 6, pp. 921942, 2009.

[88] L. Chen, D. J. Marble, R. Agha et al., "The progression of inflammation parallels the dermal angiogenesis in a keratin 14 IL-4-transgenic model of atopic dermatitis," Microcirculation, vol. 15, no. 1, pp. 49-64, 2008.

[89] M. Valko-Rokytovska, K. Bruchata, J. Simkova, M. Milkovicova, and Z. Kostecka, "Current trends in the treatment of malignant melanoma," Neoplasma, vol. 63, no. 3, pp. 333-341, 2016.

[90] D. Klinac, E. S. Gray, M. Millward, and M. Ziman, "Advances in personalized targeted treatment of metastatic melanoma and non-invasive tumor monitoring," Frontiers in Oncology, vol. 3, p. 54, 2013.

[91] C. Ferreli, G. Gasparini, A. Parodi, E. Cozzani, F. Rongioletti, and L. Atzori, "Cutaneous manifestations of scleroderma and scleroderma-like disorders: a comprehensive review," Clinical Reviews in Allergy and Immunology, vol. 53, no. 3, pp. 306-336, 2017.

[92] D. S. Rigel, J. Russak, and R. Friedman, "The evolution of melanoma diagnosis: 25 years beyond the ABCDs," CA: A Cancer Journal for Clinicians, vol. 60, no. 5, pp. 301-316, 2010.

[93] J. P. Fruehauf and V. Trapp, "Reactive oxygen species: an Achilles' heel of melanoma?," Expert Review of Anticancer Therapy, vol. 8, no. 11, pp. 1751-1757, 2008.

[94] M. Ricote and C. Glass, "PPARs and molecular mechanisms of transrepression," Biochimica et Biophysica Acta (BBA) Molecular and Cell Biology of Lipids, vol. 1771, no. 8, pp. 926-935, 2007.

[95] D. E. Hu and K. M. Brindle, "Immune cell-induced synthesis of NO and reactive oxygen species in lymphoma cells causes their death by apoptosis," FEBS Letters, vol. 579, no. 13, pp. 2833-2841, 2005.

[96] U. Leiter, R. M. Schmid, P. Kaskel, R. U. Peter, and G. Krahn, "Antiapoptotic bcl-2 and bcl-xL in advanced malignant melanoma," Archives of Dermatological Research, vol. 292, no. 5, pp. 225-232, 2000.

[97] M. Venza, M. Visalli, C. Beninati, G. V. De Gaetano, D. Teti, and I. Venza, "Cellular mechanisms of oxidative 
stress and action in melanoma," Oxidative Medicine and Cellular Longevity, vol. 2015, Article ID 481782, 11 pages, 2015.

[98] E. Obrador, F. Liu-Smith, R. W. Dellinger, R. Salvador, F. L. Meyskens, and J. M. Estrela, "Oxidative stress and antioxidants in the pathophysiology of malignant melanoma," Biological Chemistry, 2018.

[99] T. Bald, T. Quast, J. Landsberg et al., "Ultraviolet-radiationinduced inflammation promotes angiotropism and metastasis in melanoma," Nature, vol. 507, no. 7490, pp. 109-113, 2014.

[100] M. Streit and M. Detmar, "Angiogenesis, lymphangiogenesis, and melanoma metastasis," Oncogene, vol. 22, no. 20, pp. 3172-3179, 2003.

[101] Y. Mizukami, W.-S. Jo, E.-M. Duerr et al., "Induction of interleukin- 8 preserves the angiogenic response in HIF- $1 \alpha-$ deficient colon cancer cells," Nature Medicine, vol. 11, no. 9, pp. 992-997, 2005.

[102] A. Sparmann and D. Bar-Sagi, "Ras-induced interleukin-8 expression plays a critical role in tumor growth and angiogenesis," Cancer Cell, vol. 6, no. 5, pp. 447-458, 2004.

[103] Y. Li, M. N. Wang, H. Li et al., "Active immunization against the vascular endothelial growth factor receptor flk1 inhibits tumor angiogenesis and metastasis," Journal of Experimental Medicine, vol. 195, no. 12, pp. 1575-1584, 2002.

[104] T. Oku, J. G. Tjuvajev, T. Miyagawa et al., "Tumor growth modulation by sense and antisense vascular endothelial growth factor gene expression: effects on angiogenesis, vascular permeability, blood volume, blood flow, fluorodeoxyglucose uptake, and proliferation of human melanoma intracerebral xenografts," Cancer Research, vol. 58, no. 18, pp. 4185-4192, 1998.

[105] B. Basu, S. Biswas, J. Wrigley, B. Sirohi, and P. Corrie, "Angiogenesis in cutaneous malignant melanoma and potential therapeutic strategies," Expert Review of Anticancer Therapy, vol. 9, no. 11, pp. 1583-1598, 2009.

[106] J. R. Nair and R. J. Moots, "Behcet's disease," Clinical Medicine, vol. 17, no. 1, pp. 71-77, 2017.

[107] E. Alpsoy, "Behçet's disease: a comprehensive review with a focus on epidemiology, etiology and clinical features, and management of mucocutaneous lesions," The Journal of Dermatology, vol. 43, no. 6, pp. 620-632, 2016.

[108] M. C. Mat, A. Sevim, I. Fresko, and Y. Tüzün, "Behçet's disease as a systemic disease," Clinics in Dermatology, vol. 32, no. 3, pp. 435-442, 2014.

[109] M. Merashli, R. E. Eid, and I. Uthman, "A review of current management of vasculo-Behcet's," Current Opinion in Rheumatology, vol. 30, no. 1, pp. 50-56, 2018.

[110] T. W. McNally, E. M. Damato, P. I. Murray, A. K. Denniston, and R. J. Barry, "An update on the use of biologic therapies in the management of uveitis in Behçet's disease: a comprehensive review," Orphanet Journal of Rare Diseases, vol. 12, no. 1, p. 130, 2017.

[111] M. Bozkurt, H. Yüksel, S. Em et al., "Serum prolidase enzyme activity and oxidative status in patients with Behçet's disease," Redox Report, vol. 19, no. 2, pp. 59-64, 2014.

[112] E. D. Sezer, K. Aksu, O. Caglayan, G. Keser, G. Karabulut, and G. Ercan, "DNA damage and its relationship with other oxidative stress parameters in Behcet's disease," Rheumatology International, vol. 32, no. 1, pp. 217-222, 2012.
[113] F. Davatchi, C. Chams-Davatchi, H. Shams et al., "Behcet's disease: epidemiology, clinical manifestations, and diagnosis," Expert Review of Clinical Immunology, vol. 13, no. 1, pp. 57-65, 2017.

[114] J. Q. Zhong, D. H. Xian, and X. Xiong, "Expression and correlation analysis of oxidative stress, Nrf 2 and HO-1 in Behcet's disease," The Chinese Journal of Dermatovenereology, vol. 30, no. 1, pp. 6-9, 2016.

[115] M. Cekmen, C. Evereklioglu, H. Er et al., "Vascular endothelial growth factor levels are increased and associated with disease activity in patients with Behçet's syndrome," International Journal of Dermatology, vol. 42, no. 11, pp. 870-875, 2003.

[116] H. Direskeneli, "Behcet's disease: infectious aetiology, new autoantigens, and HLA-B51," Annals of the Rheumatic Diseases, vol. 60, no. 11, pp. 996-1002, 2001.

[117] K. Aksu, A. Donmez, and G. Keser, "Inflammation-induced thrombosis: mechanisms, disease associations and management," Current Pharmaceutical Design, vol. 18, no. 11, pp. 1478-1493, 2012.

[118] Z. Habibagahi, M. Habibagahi, and M. Heidari, "Raised concentration of soluble form of vascular endothelial cadherin and IL-23 in sera of patients with Behçet's disease," Modern Rheumatology, vol. 20, no. 2, pp. 154-159, 2010.

[119] E. Bozoglu, A. Dinc, H. Erdem, S. Pay, I.Simsek, and I. H. Kocar, "Vascular endothelial growth factor and monocyte chemoattractant protein-1 in Behçet's patients with venous thrombosis," Clinical and Experimental Rheumatology, vol. 23, no. 4, pp. 42-48, 2005.

[120] B. Yalcin, N. Arda, G. G. Tezel, M.Erman, and N. Alli, "Expressions of vascular endothelial growth factor and CD34 in oral aphthous lesions of Behçet's disease," Analytical and Quantitative Cytology and Histology, vol. 28, no. 6, pp. 303-306, 2006.

[121] M. L. Wahl, T. Moser, and S. V. Pizzo, "Angiostatin and antiangiogenic therapy in human disease," Recent Progress in Hormone Research, vol. 59, no. 1, pp. 73-104, 2004.

[122] M. Kamoun, M. H. Houman, A. Hamzaoui, and K. Hamzaoui, "Vascular endothelial growth factor gene polymorphisms and serum levels in Behçet's disease," Tissue Antigens, vol. 72, no. 6, pp. 581-587, 2008.

[123] T. M. Frech, V. K. Shanmugam, A. A. Shah et al., "Treatment of early diffuse systemic sclerosis skin disease," Clinical and Experimental Rheumatology, vol. 31, pp. 166-171, 2013.

[124] S. Chaowattanapanit, C. Choonhakarn, C. Foocharoen, and N. Julanon, "Phototherapy in systemic sclerosis: review," Photodermatology, Photoimmunology \& Photomedicine, vol. 33, no. 6, pp. 296-305, 2017.

[125] R. Vona, A. Giovannetti, L. Gambardella, W. Malorni, D. Pietraforte, and E. Straface, "Oxidative stress in the pathogenesis of systemic scleroderma: an overview," Journal of Cellular and Molecular Medicine, vol. 22, no. 7, pp. 3308-3314, 2018.

[126] D. F. Murrell, "A radical proposal for the pathogenesis of scleroderma," Journal of the American Academy of Dermatology, vol. 28, no. 1, pp. 78-85, 1993.

[127] B. Grygiel-Górniak and M. Puszczewicz, "Oxidative damage and antioxidative therapy in systemic sclerosis," Mediators of Inflammation, vol. 2014, Article ID 389582, 11 pages, 2014.

[128] H. Dosoki, A. Stegemann, M. Taha et al., "Targeting of $\mathrm{NADPH}$ oxidase in vitro and in vivo suppresses fibroblast 
activation and experimental skin fibrosis," Experimental Dermatology, vol. 26, no. 1, pp. 73-81, 2017.

[129] M. J. Mulligan-Kehoe and M. Simons, "Vascular disease in scleroderma: angiogenesis and vascular repair," Rheumatic Diseases Clinics of North America, vol. 34, no. 1, pp. 73-79, 2008.

[130] L. K. Hummers, A. Hall, F. M. Wigley, and M. Simons, "Abnormalities in the regulators of angiogenesis in patients with scleroderma," The Journal of Rheumatology, vol. 36, no. 3, pp. 576-582, 2009.

[131] M. Mittag, P. Beckheinrich, and U. F. Haustein, "Systemic sclerosis-related Raynaud's phenomenon: effects of iloprost infusion therapy on serum cytokine, growth factor and soluble adhesion molecule levels," Acta Dermato-Venereologica, vol. 81, no. 4, pp. 294-297, 2001.

[132] O. Distler, A. del Rosso, R. Giacomelli et al., “Angiogenic and angiostatic factors in systemic sclerosis: increased levels of vascular endothelial growth factor are a feature of the earliest disease stages and are associated with the absence of fingertip ulcers," Arthritis Research, vol. 4, no. 6, article R11, 2002.

[133] J. J. Choi, D. J. Min, M. L. Cho et al., "Elevated vascular endothelial growth factor in systemic sclerosis," The Journal of Rheumatology, vol. 30, no. 7, pp. 1529-1533, 2003.

[134] C. A. Davies, M. Jeziorska, A. J. Freemont, and A. L. Herrick, "The differential expression of VEGF, VEGFR-2, and GLUT1 proteins in disease subtypes of systemic sclerosis," Human Pathology, vol. 37, no. 2, pp. 190-197, 2006.

[135] Z. Mackiewicz, A. Sukura, D. Povilenaité et al., "Increased but imbalanced expression of VEGF and its receptors has no positive effect on angiogenesis in systemic sclerosis skin," Clinical and Experimental Rheumatology, vol. 20, no. 5, pp. 641646, 2002.

[136] O. Distler, J. H. W. Distler, A. Scheid et al., "Uncontrolled expression of vascular endothelial growth factor and its receptors leads to insufficient skin angiogenesis in patients with systemic sclerosis," Circulation Research, vol. 95, no. 1, pp. 109-116, 2004.

[137] E. J. van Zuuren, "Rosacea," The New England Journal of Medicine, vol. 377, no. 18, pp. 1754-1764, 2017.

[138] F. Anzengruber, J. Czernielewski, C. Conrad et al., "Swiss S1 guideline for the treatment of rosacea," Journal of the European Academy of Dermatology and Venereology, vol. 31, no. 11, pp. 1775-1791, 2017.

[139] A. M. Two, W. Wu, R. L. Gallo, and T. R. Hata, "Rosacea: part II. Topical and systemic therapies in the treatment of rosacea," Journal of the American Academy of Dermatology, vol. 72, no. 5, pp. 761-770, 2015.

[140] Z. Takci, S. G. Bilgili, A. S. Karadag, M. E. Kucukoglu, S. Selek, and M. Aslan, "Decreased serum paraoxonase and arylesterase activities in patients with rosacea," Journal of the European Academy of Dermatology and Venereology, vol. 29, no. 2, pp. 367-370, 2015.

[141] V. S. Tisma, A. Basta-Juzbasic, M. Jaganjac et al., "Oxidative stress and ferritin expression in the skin of patients with rosacea," Journal of the American Academy of Dermatology, vol. 60, no. 2, pp. 270-276, 2009.

[142] Y. R. Woo, J. H. Lim, D. H. Cho, and H. J. Park, "Rosacea: molecular mechanisms and management of a chronic cutaneous inflammatory condition," International Journal of Molecular Sciences, vol. 17, no. 9, 2016.
[143] J. T. M. Bamford, "Rosacea: current thoughts on origin," Seminars in Cutaneous Medicine and Surgery, vol. 20, no. 3, pp. 199-206, 2001.

[144] K. Aroni, E. Tsagroni, N. Kavantzas, E. Patsouris, and E. Ioannidis, "A study of the pathogenesis of rosacea: how angiogenesis and mast cells may participate in a complex multifactorial process," Archives of Dermatological Research, vol. 300, no. 3, pp. 125-131, 2008.

[145] M. Kim, K.-E. Kim, H. Y. Jung et al., "Recombinant erythroid differentiation regulator 1 inhibits both inflammation and angiogenesis in a mouse model of rosacea," Experimental Dermatology, vol. 24, no. 9, pp. 680-685, 2015.

[146] A. H. A. Gomaa, M. Yaar, M. M. K. Eyada, and J. Bhawan, "Lymphangiogenesis and angiogenesis in non-phymatous rosacea," Journal of Cutaneous Pathology, vol. 34, no. 10, pp. 748-753, 2007.

[147] S. V. Trufanov and N. P. Shakhbazyan, "Ophthalmic rosacea: etiopathogenesis and modern treatment methods," Vestnik oftal'mologii, vol. 134, no. 3, pp. 121-128, 2018.

[148] P. Cuevas and J. M. Arrazola, "Therapeutic response of rosacea to dobesilate," European Journal of Medical Research, vol. 10, no. 10, pp. 454-456, 2005.

[149] R. K. Jain, D. G. Duda, J. W. Clark, and J. S. Loeffler, "Lessons from phase III clinical trials on anti-VEGF therapy for cancer," Nature Clinical Practice Oncology, vol. 3, no. 1, pp. 2440, 2006.

[150] D. G. Duda, T. T. Batchelor, C. G. Willett, and R. K. Jain, "VEGF-targeted cancer therapy strategies: current progress, hurdles and future prospects," Trends in Molecular Medicine, vol. 13, no. 6, pp. 223-230, 2007.

[151] M. Chan, K. Sjoquist, and J. Zalcberg, "Clinical utility of ramucirumab in advanced gastric cancer," Biologics: Targets and Therapy, vol. 9, pp. 93-105, 2015.

[152] C. Y. Ng, H. Yen, H. Y. Hsiao, and S. C. Su, "Phytochemicals in skin cancer prevention and treatment: an updated review," International Journal of Molecular Sciences, vol. 19, no. 4, 2018.

[153] B. Renganathan, V. Durairaj, D. C. Kirman, P. K. A. Esubonteng, S. K. Ang, and R. Ge, "Recombinant TSR1 of ADAMTS5 suppresses melanoma growth in mice via an anti-angiogenic mechanism," Cancers, vol. 10, no. 6, 2018.

[154] E. Dupont, P. E. Savard, C. Jourdain et al., "Antiangiogenic properties of a novel shark cartilage extract: potential role in the treatment of psoriasis," Journal of Cutaneous Medicine and Surgery, vol. 2, no. 3, pp. 146-152, 1998.

[155] A. H. Norooznezhad and F. Norooznezhad, "Cannabinoids: possible agents for treatment of psoriasis via suppression of angiogenesis and inflammation," Medical Hypotheses, vol. 99, pp. 15-18, 2017.

[156] Y. H. Kuang, Y. Lu, Y. K. Liu et al., "Topical sunitinib ointment alleviates psoriasis-like inflammation by inhibiting the proliferation and apoptosis of keratinocytes," European Journal of Pharmacology, vol. 824, pp. 57-63, 2018.

[157] N. Maruotti, F. P. Cantatore, and D. Ribatti, “Thalidomide in treatment of connective diseases and vasculities," Reumatismo, vol. 58, no. 3, pp. 187-190, 2006.

[158] P. Li, Y. Li, H. Jiang et al., "Glabridin, an isoflavan from licorice root, ameliorates imiquimod-induced psoriasis-like inflammation of BALB/c mice," International Immunopharmacology, vol. 59, pp. 243-251, 2018. 
[159] S. Korkmaz, I. Erturan, M. Nazıroğlu, A. C. Uğuz, B. Ciğ, and I. S. Övey, "Colchicine modulates oxidative stress in serum and neutrophil of patients with Behçet disease through regulation of $\mathrm{Ca}^{2+}$ release and antioxidant system," The Journal of Membrane Biology, vol. 244, no. 3, pp. 113-120, 2011.

[160] K. Wang, H. Jiang, W. Li, M. Qiang, T. Dong, and H. Li, "Role of vitamin C in skin diseases," Frontiers in Physiology, vol. 9, p. 891, 2018.

[161] S. Byun, E. Lee, Y. J. Jang, Y. Kim, and K. W. Lee, "The NADPH oxidase inhibitor apocynin inhibits UVB-induced skin carcinogenesis," Experimental Dermatology, vol. 25, no. 6, pp. 489-491, 2016.

[162] P. Sharma, M. K. Montes de Oca, A. R. Alkeswani et al., "Tea polyphenols for the prevention of UVB-induced skin cancer," Photodermatology, Photoimmunology \& Photomedicine, vol. 34, no. 1, pp. 50-59, 2018.

[163] S. Zhang, X. Liu, L. Mei, H. Wang, and F. Fang, "Epigallocatechin-3-gallate (EGCG) inhibits imiquimod-induced psoriasis-like inflammation of BALB/c mice," BMC Complementary and Alternative Medicine, vol. 16, no. 1, p. 334, 2016.

[164] S. Li, M. Xu, Q. Niu et al., "Efficacy of procyanidins against in vivo cellular oxidative damage: a systematic review and meta-analysis," PLoS One, vol. 10, no. 10, article e0139455, 2015.

[165] R. R. Bansode, J. R. Khatiwada, J. N. Losso, and L. L. Williams, "Targeting microRNA in cancer using plant-based proanthocyanidins," Diseases, vol. 4, no. 4, 2016.

[166] M. Działo, J. Mierziak, U. Korzun, M. Preisner, J. Szopa, and A. Kulma, "The potential of plant phenolics in prevention and therapy of skin disorders," International Journal of Molecular Sciences, vol. 17, no. 2, p. 160, 2016.

[167] R. Lai, D. Xian, X. Xiong, L. Yang, J. Song, and J. Zhong, "Proanthocyanidins: novel treatment for psoriasis that reduces oxidative stress and modulates Th17 and Treg cells," Redox Report, vol. 23, no. 1, pp. 130-135, 2018.

[168] J. Zhong, D. Xian, X. Xiong, and J. Liu, "Oral allicin in the treatment of Behcet's disease through attenuating oxidative stress: a pilot study in 20patients with mucocutaneous lesions," Dermatologic Therapy, vol. 30, no. 1, pp. 1-4, 2017. 


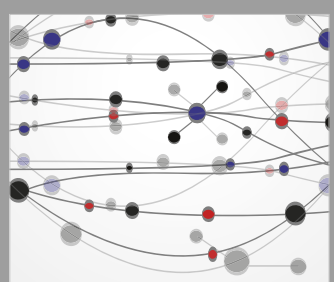

The Scientific World Journal
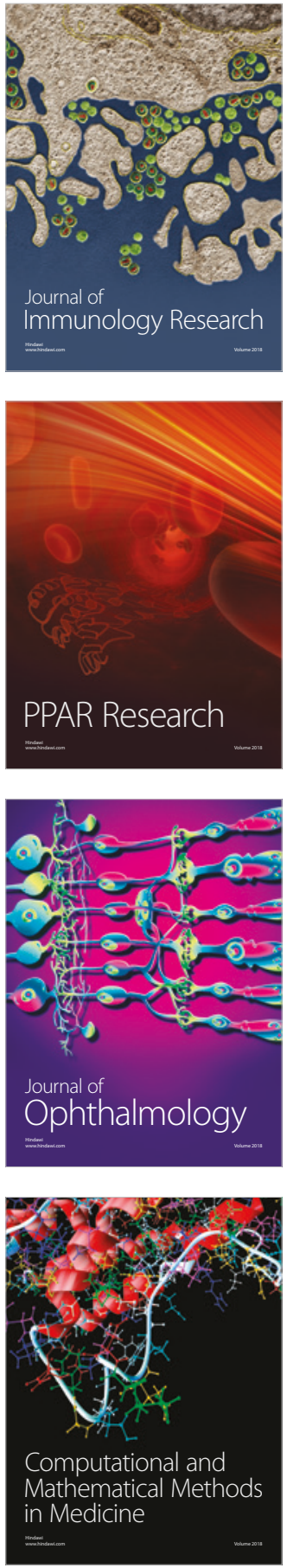

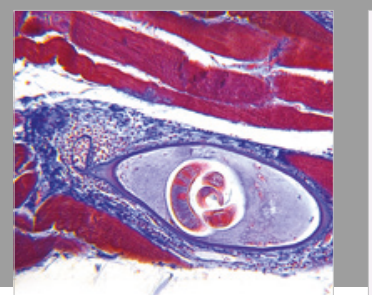

Gastroenterology Research and Practice

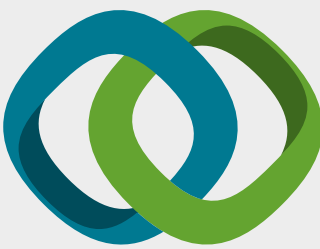

\section{Hindawi}

Submit your manuscripts at

www.hindawi.com
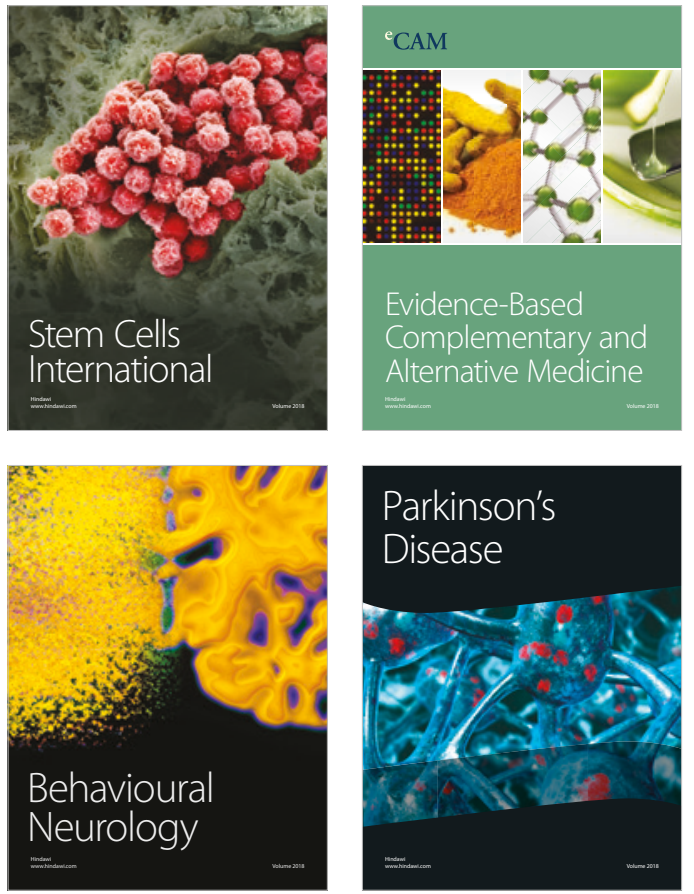

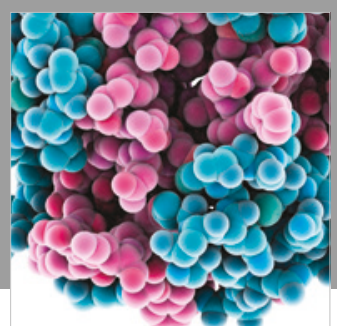

ournal of

Diabetes Research

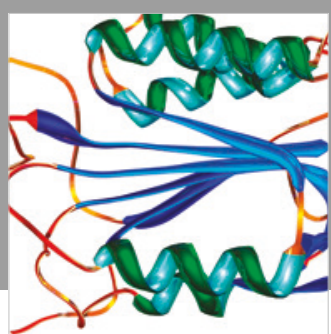

Disease Markers
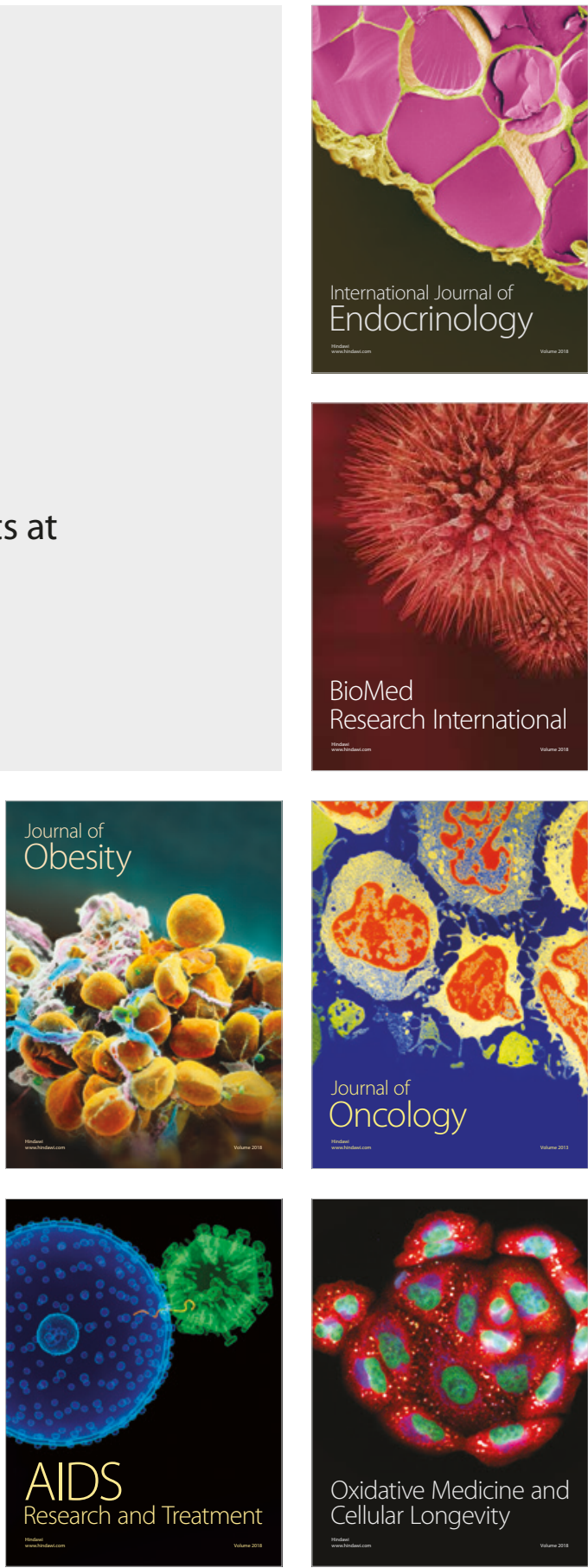